\title{
MAGNETIC FIELD EXPANSION IN A BENT-SOLENOID CHANNEL*
}

\author{
Chun-xi Wang ${ }^{\dagger}$ and Lee C. Teng \\ Advanced Photon Source, Argonne National Laboratory, Argonne, IL 60439
}

\begin{abstract}
Bent-solenoid channels are being considered for generating dispersions desired for emittance exchange between the transverse and longitudinal motions of a muon beam in a longitudinal ionization cooling channel. Correct field expansion around the reference orbit is important for reliable particle-tracking simulation as well as beam dynamics analysis. This paper reviews the magnetic field expansion for a bent-solenoid channel. In particular, ready-to-use formulas for the magnetic field and its potentials are given.
\end{abstract}

\section{INTRODUCTION}

Bent-solenoid channels are being studied for longitudinal ionization cooling of a muon beam [1] in which the strong longitudinal magnetic field from the solenoid provides transverse focusing and the superimposed transverse dipole field provides dispersions desired for emittance exchange. In addition, a quadrupole field may be superimposed, for example, to achieve symmetric focusing. To study the beam dynamics in a bent-solenoid channel, magnetic field expansion around the reference orbit is needed. Although general formalism for such expansion is wellestablished, a reliable, ready-to-use result for bent solenoid seems not available. Furthermore, many errors, from "typos" to technical mistakes, exist in the literature on field expansion, as well as in computer code currently in use. In this paper, following the approach presented in ref. [2], we briefly review the derivation of field expansion and present ready-to-use expressions for the magnetic field and the corresponding scaler and vector potentials up to the octupole order.

\section{GENERAL METHOD}

The static magnetic field $\mathbf{B}$ in a beam pipe must satisfy the source-free Maxwell equations in vacuum $\nabla \cdot \mathbf{B}=0$ and $\nabla \times \mathbf{B}=0$. It is well known that both a scaler potential $\phi$ and a vector potential $\mathbf{A}$ can be defined such that $\mathbf{B}=$ $\nabla \times \mathbf{A}$ and $\mathbf{B}=-\nabla \phi$. To study particle transport in a beam line, it is necessary to have a general field expression that depends on free parameters yet satisfies Maxwell equations in the neighborhood of a given design orbit.

\section{$2.1 \quad$ Frenet-Serret Coordinate System}

The Frenet-Serret coordinate system is commonly used to describe a particle's motion around a design orbit. Its

\footnotetext{
${ }^{*}$ Work supported by the U.S. Department of Energy, Office of Basic Energy Sciences, under Contract No. W-31-109-ENG-38.

$\dagger$ Wangcx@aps.anl.gov
}

base vectors are defined by the unit vectors $\left(\mathbf{e}_{s}, \mathbf{e}_{x}, \mathbf{e}_{y}\right)$ of the moving trihedron that can be determined from the Frenet formulas

$$
\mathbf{e}_{s}^{\prime}=-\kappa \mathbf{e}_{x}, \mathbf{e}_{x}^{\prime}=\kappa \mathbf{e}_{s}+\tau \mathbf{e}_{y}, \mathbf{e}_{y}^{\prime}=-\tau \mathbf{e}_{x},
$$

where the prime indicates derivative with respect to the path length $s$ along the reference orbit, and $\kappa(s)$ and $\tau(s)$ are the local curvature and torsion of the reference orbit. (Note that, $\mathbf{e}_{x}$ and $\mathbf{e}_{y}$ are the opposites of the principal normal and binormal vectors commonly used in curve theory.) We consider the most common case that the reference orbit is a well-behaved plane (torsion-free) curve: $\tau(s)=0$.

In the Frenet-Serret coordinate system, a particle's position $\mathbf{r}(s)$ can be written as

$$
\mathbf{r}(s)=\mathbf{r}_{0}(s)+x \mathbf{e}_{x}+y \mathbf{e}_{y},
$$

where $\mathbf{r}_{0}(s)$ is the reference orbit, and $x$ and $y$ are the transverse displacements from it. A vector field $\mathbf{A}(x, y, s)$ can be decomposed as

$$
\mathbf{A}=A_{x} \mathbf{e}_{x}+A_{y} \mathbf{e}_{y}+A_{s} \mathbf{e}_{s} .
$$

Note that, in the literature, $A_{s}$ may represent the canonical component $h \mathbf{A} \cdot \mathbf{e}_{s}$ instead of $\mathbf{A} \cdot \mathbf{e}_{s}$.

Vector analysis using the Frenet-Serret coordinates can be carried out via

$$
\begin{aligned}
\nabla \phi & =\frac{\partial \phi}{\partial x} \mathbf{e}_{x}+\frac{\partial \phi}{\partial y} \mathbf{e}_{y}+\frac{1}{h} \frac{\partial \phi}{\partial s} \mathbf{e}_{s} \\
\nabla \cdot \mathbf{A} & =\frac{1}{h} \frac{\partial\left(h A_{x}\right)}{\partial x}+\frac{\partial A_{y}}{\partial y}+\frac{1}{h} \frac{\partial A_{s}}{\partial s} \\
\nabla \times \mathbf{A} & =\left[\frac{\partial A_{s}}{\partial y}-\frac{1}{h} \frac{\partial A_{y}}{\partial s}\right] \mathbf{e}_{x}+\frac{1}{h}\left[\frac{\partial A_{x}}{\partial s}-\frac{\partial\left(h A_{s}\right)}{\partial x}\right] \mathbf{e}_{y} \\
& +\left[\frac{\partial A_{y}}{\partial x}-\frac{\partial A_{x}}{\partial y}\right] \mathbf{e}_{s}
\end{aligned}
$$

where the so-called scale factor, $h=1+\kappa(s) x$.

\subsection{Scaler Potential and Recursion Formula}

Expanding the magnetic field and its potentials as a series of $x$ and $y$, a general magnetic field can be specified by the expansion coefficients as functions of $s$. Because the field must satisfy the Maxwell equations, those expansion coefficients are interrelated. An important exercise is to identify coefficients that can be chosen freely and express the field as a function of them.

It is convenient to start with the scaler potential $\phi(x, y, s)$. Using the Taylor series, $\phi$ can be expanded as

$$
\phi(x, y, s)=-\sum_{n=0}^{\infty} \sum_{m=0}^{n} a_{n-m, m}(s) \frac{x^{n-m}}{(n-m) !} \frac{y^{m}}{m !} \text {. }
$$


The Maxwell equations are satisfied if and only if the scaler potential satisfies the Laplace equation $\nabla^{2} \phi=0$. Working out $\nabla^{2} \phi$ using Eqs. $(7,4,5)$ and expanding the result into a Taylor series of $x$ and $y$, then requiring the coefficients to be zero, gives the recursive formula that the coefficient functions must satisfy

$$
\begin{gathered}
a_{n, m+2}=-a_{n+2, m}-\sum_{p=0}^{n}(-\kappa)^{p} \frac{n !}{(n-p) !}\left[\kappa a_{n-p+1, m}\right. \\
\left.+(p+1) a_{n-p, m}^{\prime \prime}-\left(\begin{array}{c}
p+2 \\
2
\end{array}\right)(n-p) \kappa^{\prime} a_{n-p-1, m}^{\prime}\right] .
\end{gathered}
$$

Although rather obscure, this formula clearly shows that, corresponding to the even and the odd $m$ 's, there are two independent sets of recursion relations for $a_{n, m}$ 's. Because the second index $m$ specifies the power of $\mathrm{y}$ in the potential expansion, the odd (even) set of coefficients defines the normal (skew) field component whose potential is antisymmetric (symmetric) to the orbit plane. Furthermore, only $a_{n, 0}(s)$ and $a_{n, 1}(s)$ can be independently chosen. All higher-order (in $m$ ) coefficients can be derived from them. Using these independent coefficients and the recursion formula, it is straightforward, though tedious, to express the scaler potential and the magnetic field as functionals of free functions that characterize the field.

\subsection{Independent Magnetic Field Components}

The transverse components of the field on the reference plane and the longitudinal component on the reference orbit are determined by the independent coefficients $a_{n, 0}$ and $a_{n, 1}$ via

$$
\begin{aligned}
\text { skew : } B_{x}(x, 0, s) & =\sum_{n=1}^{\infty} a_{n, 0}(s) \frac{x^{n-1}}{(n-1) !}, \\
\text { normal : } B_{y}(x, 0, s) & =\sum_{n=0}^{\infty} a_{n, 1}(s) \frac{x^{n}}{n !}, \\
\text { longitudinal : } B_{s}(0,0, s) & =a_{0,0}^{\prime}(s) .
\end{aligned}
$$

Conversely, these three field components completely determine the independent coefficients and the 3D magnetic field. Furthermore, these field components can be freely chosen.

In terms of standard (US convention) multipole coefficients $a_{n}$ and $b_{n}$ defined by [3]

$$
B_{y}+i B_{x}=\sum_{n=0}^{\infty}\left(b_{n}+i a_{n}\right)(x+i y)^{n},
$$

the independent coefficients of the transverse field are

$$
\begin{aligned}
& a_{n, 1}=\left.\frac{\partial^{n} B_{y}}{\partial x^{n}}\right|_{x=y=0}=n ! b_{n}, \\
& a_{n, 0}=\left.\frac{\partial^{n-1} B_{x}}{\partial x^{n-1}}\right|_{x=y=0}=(n-1) ! a_{n-1}, \\
& a_{0,0}^{\prime} \equiv b_{s}
\end{aligned}
$$

for the normal, skew, and longitudinal components, respectively. Note that we used the absolute multipole strengths instead of normalizing them by the dipole strength $B_{0}$. Up to the octupole order, the independent coefficient functions are $b_{s}(s)$ for the solenoid component, $b_{0}(s)$ for the dipole, $b_{1}(s)$ and $a_{1}(s)$ for the normal and skew quadrupoles, $b_{2}(s)$ and $a_{2}(s)$ for the sextupoles, and $b_{3}(s)$ and $a_{3}(s)$ for the octupoles.

\subsection{Vector Potential}

The vector potential $\mathbf{A}$ is important in Hamiltonian treatment of beam dynamics. To obtain a unique expression of the vector potential from the scaler potential $\phi$, we choose the gauge

$$
x A_{x}+y A_{y}=0
$$

instead of the usual Coulomb gauge $\nabla \cdot \mathbf{A}=0$. Using this gauge, we can introduce two new functions, $F(x, y, s)$ and $G(x, y, s)$, to express the three components of the vector potential as

$$
A_{x}=-y F, \quad A_{y}=x F, \quad A_{s}=\frac{G}{h} .
$$

Then the magnetic field $\mathbf{B}=\nabla \times \mathbf{A}$ becomes

$$
\begin{aligned}
B_{x} & =\frac{1}{h}\left(\frac{\partial G}{\partial y}-x \frac{\partial F}{\partial s}\right) \\
B_{y} & =\frac{1}{h}\left(-\frac{\partial G}{\partial x}-y \frac{\partial F}{\partial s}\right) \\
B_{s} & =x \frac{\partial F}{\partial x}+y \frac{\partial F}{\partial y}+2 F
\end{aligned}
$$

or

$$
\begin{aligned}
& x \frac{\partial G}{\partial x}+y \frac{\partial G}{\partial y}=h\left(y B_{x}-x B_{y}\right), \\
& x \frac{\partial F}{\partial x}+y \frac{\partial F}{\partial y}+2 F=B_{s} .
\end{aligned}
$$

The equations for $G$ and $F$ are decoupled and can be solved separately via Euler's theorem for homogenous forms. It is easy to verify that their solutions can be written as

$$
\begin{aligned}
F & =\sum_{n=0}^{\infty} \frac{1}{n+2} B_{s}^{(n)}, \\
G & =\sum_{n=1}^{\infty}\left(\frac{1}{n}+\frac{\kappa x}{n+1}\right)\left(y B_{x}^{(n-1)}-x B_{y}^{(n-1)}\right) .
\end{aligned}
$$

Here the superscript $(n)$ means the $n$th order component. Using the $F$ and $G$ expressions and Eq. (17), the vector potential can be straightforwardly worked out from the magnetic field expansion. Here we will not spell out the expressions as functions of $a_{n, m}$. (Note that the last expression for $F$ in ref. [2] is incorrect.) 


\section{READY-TO-USE FIELD EXPANSION}

In the previous section, we outlined the procedure to obtain the Taylor expansion of a magnetic field and its scaler and vector potentials. Although straightforward in principle, it is a rather tedious and fallible exercise to obtain the final expansions, especially with the dependent coefficients removed. With the help of Mathematica, we did this exercise for the bent-solenoid channels considered for the ionization cooling of a muon beam. We report the readyto-use formulas that include all field components up to the octupole order, except for the horizontal dipole component $a_{0}(s)$, which makes the zeroth-order orbit a nonplanar curve. If the zeroth-order orbit is chosen as a reference, $\kappa(s)=q b_{0}(s) / p_{s}$, where $q$ and $p_{s}$ are the charge and longitudinal momentum. The bending radius $\rho(s)=|1 / \kappa|$.

$$
\begin{aligned}
& \phi(x, y, s)=a_{0,0}+b_{0} y \\
& +\frac{1}{2} a_{1} x^{2}+b_{1} x y-\frac{1}{2}\left(a_{1}+b_{s}^{\prime}\right) y^{2} \\
& +\frac{1}{3} a_{2} x^{3}+b_{2} x^{2} y-\frac{1}{2}\left[2 a_{2}+\kappa\left(a_{1}-2 b_{s}^{\prime}\right)-\kappa^{\prime} b_{s}\right] x y^{2} \\
& -\frac{1}{6}\left(2 b_{2}+b_{0}^{\prime \prime}+\kappa b_{1}\right) y^{3}+\frac{1}{4} a_{3} x^{4}+b_{3} x^{3} y \\
& -\frac{1}{4}\left[6 a_{3}+a_{1}^{\prime \prime}+2 \kappa\left(a_{2}+3 \kappa^{\prime} b_{s}\right)-2 \kappa^{2}\left(a_{1}-3 b_{s}^{\prime}\right)\right] x^{2} y^{2} \\
& -\frac{1}{6}\left[6 b_{3}+b_{1}^{\prime \prime}+2 \kappa\left(b_{2}-b_{0}^{\prime \prime}\right)-\kappa^{2} b_{1}-\kappa^{\prime} b_{0}^{\prime}\right] x y^{3} \\
& +\frac{1}{24}\left[6 a_{3}+2 a_{1}^{\prime \prime}+b_{s}^{\prime \prime \prime}+\kappa\left(4 a_{2}+5 \kappa^{\prime} b_{s}\right)-\kappa^{2}\left(a_{1}-4 b_{s}^{\prime}\right)\right] y^{4}
\end{aligned}
$$$$
B_{x}(x, y, s)=a_{1} x+b_{1} y+a_{2} x^{2}+2 b_{2} x y
$$$$
-\frac{1}{2}\left[2 a_{2}+\kappa\left(a_{1}-2 b_{s}^{\prime}\right)-\kappa^{\prime} b_{s}\right] y^{2}+a_{3} x^{3}+3 b_{3} x^{2} y
$$$$
-\frac{1}{2}\left[6 a_{3}+a_{1}^{\prime \prime}+2 \kappa\left(a_{2}+3 \kappa^{\prime} b_{s}\right)-2 \kappa^{2}\left(a_{1}-3 b_{s}^{\prime}\right)\right] x y^{2}
$$$$
-\frac{1}{6}\left[6 b_{3}+b_{1}^{\prime \prime}+2 \kappa\left(b_{2}-b_{0}^{\prime \prime}\right)-\kappa^{2} b_{1}-\kappa^{\prime} b_{0}^{\prime}\right] y^{3}
$$$$
B_{y}(x, y, s)=b_{0}+b_{1} x-\left(a_{1}+b_{s}^{\prime}\right) y
$$$$
+b_{2} x^{2}-\left[2 a_{2}+\kappa\left(a_{1}-2 b_{s}^{\prime}\right)-\kappa^{\prime} b_{s}\right] x y
$$$$
-\frac{1}{2}\left(2 b_{2}+b_{0}^{\prime \prime}+\kappa b_{1}\right) y^{2}+b_{3} x^{3}
$$$$
-\frac{1}{2}\left[6 a_{3}+a_{1}^{\prime \prime}+2 \kappa\left(a_{2}+3 \kappa^{\prime} b_{s}\right)-2 \kappa^{2}\left(a_{1}-3 b_{s}^{\prime}\right)\right] x^{2} y
$$$$
-\frac{1}{2}\left[6 b_{3}+b_{1}^{\prime \prime}+2 \kappa\left(b_{2}-b_{0}^{\prime \prime}\right)-\kappa^{2} b_{1}-\kappa^{\prime} b_{0}^{\prime}\right] x y^{2}
$$$$
+\frac{1}{6}\left[6 a_{3}+2 a_{1}^{\prime \prime}+b_{s}^{\prime \prime \prime}+\kappa\left(4 a_{2}+5 \kappa^{\prime} b_{s}\right)-\kappa^{2}\left(a_{1}-4 b_{s}^{\prime}\right)\right] y^{3}
$$$$
B_{s}(x, y, s)=b_{s}-\kappa b_{s} x+b_{0}^{\prime} y
$$$$
+\frac{1}{2}\left(a_{1}^{\prime}+2 \kappa^{2} b_{s}\right) x^{2}+\left(b_{1}^{\prime}-\kappa b_{0}^{\prime}\right) x y-\frac{1}{2}\left(a_{1}^{\prime}+b_{s}^{\prime \prime}\right) y^{2}
$$$$
+\frac{1}{6}\left(2 a_{2}^{\prime}-3 \kappa a_{1}^{\prime}-6 \kappa^{3} b_{s}\right) x^{3}+\left(b_{2}^{\prime}-\kappa b_{1}^{\prime}+\kappa^{2} b_{0}^{\prime}\right) x^{2} y
$$

$$
\begin{aligned}
& -\frac{1}{2}\left[2 a_{2}^{\prime}-3 \kappa b_{s}^{\prime \prime}+\kappa^{\prime}\left(a_{1}-3 b_{s}^{\prime}\right)-\kappa^{\prime \prime} b_{s}\right] x y^{2} \\
& -\frac{1}{6}\left(2 b_{2}^{\prime}+b_{0}^{\prime \prime \prime}+\kappa b_{1}^{\prime}+\kappa^{\prime} b_{1}\right) y^{3}
\end{aligned}
$$

$A_{x}(x, y, s)=-\frac{1}{2} b_{s} y+\frac{1}{3} \kappa b_{s} x y-\frac{1}{3} b_{0}^{\prime} y^{2}$

$-\frac{1}{8}\left(2 \kappa^{2} b_{s}+a_{1}^{\prime}\right) x^{2} y-\frac{1}{4}\left(b_{1}^{\prime}-\kappa b_{0}^{\prime}\right) x y^{2}+\frac{1}{8}\left(a_{1}^{\prime}+b_{s}^{\prime \prime}\right) y^{3}$

$-\frac{1}{30}\left(2 a_{2}^{\prime}-3 \kappa a_{1}^{\prime}-6 \kappa^{3} b_{s}\right) x^{3} y-\frac{1}{5}\left(b_{2}^{\prime}-\kappa b_{1}^{\prime}+\kappa^{2} b_{0}^{\prime}\right) x^{2} y^{2}$

$+\frac{1}{10}\left[2 a_{2}^{\prime}-3 \kappa b_{s}^{\prime \prime}+\kappa^{\prime}\left(a_{1}-3 b_{s}^{\prime}\right)-\kappa^{\prime \prime} b_{s}\right] x y^{3}$

$+\frac{1}{30}\left(2 b_{2}^{\prime}+b_{0}^{\prime \prime \prime}+\kappa b_{1}^{\prime}+\kappa^{\prime} b_{1}\right) y^{4}$

$A_{y}(x, y, s)=\frac{1}{2} b_{s} x-\frac{1}{3} \kappa b_{s} x^{2}+\frac{1}{3} b_{0}^{\prime} x y$

$+\frac{1}{8}\left(2 \kappa^{2} b_{s}+a_{1}^{\prime}\right) x^{3}+\frac{1}{4}\left(b_{1}^{\prime}-\kappa b_{0}^{\prime}\right) x^{2} y-\frac{1}{8}\left(a_{1}^{\prime}+b_{s}^{\prime \prime}\right) x y^{2}$

$+\frac{1}{30}\left(2 a_{2}^{\prime}-3 \kappa a_{1}^{\prime}-6 \kappa^{3} b_{s}\right) x^{4}+\frac{1}{5}\left(b_{2}^{\prime}-\kappa b_{1}^{\prime}+\kappa^{2} b_{0}^{\prime}\right) x^{3} y$

$-\frac{1}{10}\left[2 a_{2}^{\prime}-3 \kappa b_{s}^{\prime \prime}+\kappa^{\prime}\left(a_{1}-3 b_{s}^{\prime}\right)-\kappa^{\prime \prime} b_{s}\right] x^{2} y^{2}$

$-\frac{1}{30}\left(2 b_{2}^{\prime}+b_{0}^{\prime \prime \prime}+\kappa b_{1}^{\prime}+\kappa^{\prime} b_{1}\right) x y^{3}$

$A_{s}(x, y, s)=-b_{0} x$

$-\frac{1}{2}\left(b_{1}-\kappa b_{0}\right) x^{2}+\frac{1}{2}\left(2 a_{1}+b_{s}^{\prime}\right) x y+\frac{1}{2} b_{1} y^{2}$

$-\frac{1}{6}\left(2 b_{2}-\kappa b_{1}+3 \kappa^{2} b_{0}\right) x^{3}+\frac{1}{6}\left(6 a_{2}-5 \kappa b_{s}^{\prime}-2 \kappa^{\prime} b_{s}\right) x^{2} y$

$+\frac{1}{6}\left(6 b_{2}+b_{0}^{\prime \prime}\right) x y^{2}-\frac{1}{6}\left[2 a_{2}+\kappa\left(a_{1}-2 b_{s}^{\prime}\right)-\kappa^{\prime} b_{s}\right] y^{3}$

$-\frac{1}{12}\left(3 b_{3}-\kappa b_{2}+2 \kappa^{2} b_{1}-6 \kappa^{3} b_{0}\right) x^{4}$

$+\frac{1}{24}\left(24 a_{3}+3 a_{1}^{\prime \prime}+26 \kappa^{2} b_{s}^{\prime}+20 \kappa \kappa^{\prime} b_{s}\right) x^{3} y$

$+\frac{1}{24}\left(36 b_{3}+3 b_{1}^{\prime \prime}-7 \kappa b_{0}^{\prime \prime}-3 \kappa^{\prime} b_{0}^{\prime}\right) x^{2} y^{2}$

$-\frac{1}{24}\left[24 a_{3}+5 a_{1}^{\prime \prime}+b_{s}^{\prime \prime \prime}+8 \kappa\left(a_{2}+3 \kappa^{\prime} b_{s}\right)\right.$

$$
\left.-8 \kappa^{2}\left(a_{1}-3 b_{s}^{\prime}\right)\right] x y^{3}
$$

$-\frac{1}{24}\left[6 b_{3}+b_{1}^{\prime \prime}+2 \kappa\left(b_{2}-b_{0}^{\prime \prime}\right)-\kappa^{2} b_{1}-\kappa^{\prime} b_{0}^{\prime}\right] y^{4}$

\section{REFERENCES}

[1] C. Ankenbradt et al., Phys. Rev. ST Accel. Beams 2, 081001 (1999).

[2] L. C. Teng, Proceedings of Particle Accelerator Conference 1995, p. 2814 (1995).

[3] A. Chao and M. Tigner (eds.), Handbook of Accelerator Physics and Engineering, World Scientific Publishing Co., p. 71 (1999). 